\title{
The addition spectrum of interacting electrons: Parametric dependence
}

\author{
Luca Bonci and Richard Berkovits \\ The Minerva Center for the Physics of Mesoscopics, Fractals and Neural Networks, \\ Department of Physics, Bar-Ilan University, Ramat-Gan 52900, Israel
}

(December 3, 2017)

\begin{abstract}
The addition spectrum of a disordered stadium is studied for up to 120 electrons using the self consistent Hartree-Fock approximation for different values of the dimensionless conductance and in the presence and absence of a neutralizing background. In all cases a Gaussian distribution of the addition spectrum is reached for $r_{s} \leq 1$. An empirical scaling for the distribution width in the presence of a neutralizing background is tested and seems to describe rather well its dependence on the dimensionless conductance.
\end{abstract}

PACS numbers: 73.23.Hk, 05.45.+b,73.20.Dx

Typeset using REVTEX 
Recent measurements of the distribution of the addition spectrum of chaotic quantum dots [1 3] differ from the results of the orthodox constant interaction (CI) model [4] in several ways. The distribution, which is roughly Gaussian in all experiments, has no bimodal structure expected due to the spin [8], nor does it have a Wigner like distribution expected if somehow the spin degeneracy is lifted [9, 10]. The width of the distribution varies between the different experiments. While in the first experiment [1] the width is considerably larger than that expected from the CI model, the width in the latest experiment [3] is compatible with the CI model predictions.

These deviations from the CI model are usually attributed to the low electronic densities of the dots. For a typical dot the electronic density is of the order of $n_{D}=2-3.5 \times 10^{11} \mathrm{~cm}^{-2}$, corresponding to a ratio of the average inter-particle Coulomb interaction and the Fermi energy $r_{s}=1 / \sqrt{\pi n_{D}} a_{B} \sim 1$ (where $a_{B}$ is the Bohr radius). At these values of $r_{s}$ correlations in the electronic densities which are not taken into account in the CI model appear [1, 10].

Thus, the study of the addition spectra for chaotic dots must take into account the intricate interplay of chaos and interaction. Several numerical studies have tried to clarify the picture using exact diagonalization methods [1,8] and Hartree-Fock (HF) approximations 11.12]. The exact diagonalization method has the advantage of taking the full effect of the correlations into account, but can treat only small systems. The HF approximations lose some of the correlation effects, but are able to handle larger systems.

In this paper we would like to answer some of the remaining open questions regarding the addition spacing distribution: (i) How does it depend on the dimensionless conductance $g$ ? (ii) Does it depend on the charge distribution in the dot (i.e., a uniform distribution due to a homogeneous background vs. accumulation of charge at the boundaries)? (iii) Do larger system sizes where up to a hundred electrons may be added to the dot change qualitatively the results obtained in the previous studies where only several electrons were added per dot?

In order to answer these questions we study the Bunimovich stadium which is a canonical example of chaotic system [13]. The addition spectrum of the stadium is calculated for different values of $g$, in the presence of a positive background and its absence, using 
a self-consistent Hartree-Fock approximation. We consider spinless electrons, since from experiment [1]3] and from our exact diagonalization calculations [8] we can argue that at $r_{s} \sim 1$ the role of spins in determining the addition spectrum is not an important one.

We find that for any value of $g$ the addition spectrum distribution is Gaussian for values of $r_{s} \sim 1$. For smaller values of $g$ the distribution becomes Gaussian at smaller values of $r_{s}$. The dependence of the distribution width on $g$ can be summed up by a scaling function given later on. For $g \rightarrow \infty$ the width grows only moderately even at $r_{s} \sim 1$, while for higher values of $g$ the distribution is much wider. We find that the background plays an important role in determining the distribution of the addition spectrum. The absence of a positive background enhances the width of the distribution due to charge accumulation on the boundaries as predicted in Ref. [9].

To perform our numerical calculations we chose a simple two dimensional tight-binding model, namely a square lattice with nearest-neighbors interaction. By using a single spatial index to label the sites, we can write the Hamiltonian as:

$$
\begin{aligned}
H & =H_{0}+H_{\text {int }} \\
H_{0} & =\sum_{j} \epsilon_{j} a_{j}^{+} a_{j}-V \sum_{<j, k>}\left(a_{j}^{+} a_{k}+a_{k}^{+} a_{j}\right) \\
H_{\text {int }} & =\sum_{j>k} U_{j k}\left(a_{j}^{+} a_{j}-K\right)\left(a_{k}^{+} a_{k}-K\right),
\end{aligned}
$$

where $\epsilon_{j}$ is the on-site energy, $V$ is the constant hopping matrix element and $\langle j, k\rangle$ denotes the sum over the nearest-neighbors. $H_{\text {int }}$ contains the interaction among the electrons, which we chose as the unscreened Coulomb interaction $U_{j k}=U /\left|r_{j}-r_{k}\right| / b$, with $b$ representing the lattice spacing and $U=e^{2} / b$ the interaction strength between electrons located on nearest-neighbor sites. Thus, $r_{s} \sim \sqrt{\pi N / n}(U / 8 V)$, where $N$ is the number of sites and $n$ the number of electrons. The constant term $K$ which appears in the interaction term represents a constant positive charge background. Setting its value equal to zero corresponds to an electrically isolated dot, while setting $K=n / N$ assures the global charge neutrality corresponding to a dot closely coupled to a gate or screened by the environment.

To describe completely the system we need also to specify the boundary conditions which 
define the shape of the dot. We studied a Bunimovich quarter stadium, see Fig. 1, to avoid degeneracies due to space symmetries. The classical motion inside this region is chaotic and the quantum dynamic shows the typical quantum chaos signatures, as the RMT statistics (avoided-level crossings and Wigner-Dyson level-spacing distribution) or the presence of quantum eigenstates scarred along the unstable classical orbits [14, 115]. This choice allowed us to study the quasi-ballistic regime of high dimensionless conductance but avoiding the highly degenerate condition related to the study of regular dots.

The addition spectrum of the dot has been studied numerically by solving the HartreeFock problem in various parameter ranges. We decoupled the Coulomb interaction in a direct and an exchange term as described in [16,17]. The Hamiltonian in the HF approximation reads

$$
H_{H F}=H_{0}+\sum_{j \neq k} a_{j}^{+} a_{j} U_{j k}\left\langle a_{k}^{+} a_{k}\right\rangle_{0}-\sum_{j \neq k} a_{j}^{+} a_{k} U_{j k}\left\langle a_{k}^{+} a_{j}\right\rangle_{0}+\text { const. }
$$

where $\langle\ldots\rangle_{0}$ denotes the average on the ground state which is calculated self-consistently.

We studied the addition spectrum by changing the number of electrons from $n=20$ to $n=120$ and repeating the calculation for 20 different realizations of the on-site energies. The dimensionless conductance of the sample is calculated from the non-interacting participation ratio $I=10^{-2} \Omega^{-1} \sum_{n=20}^{120} \sum_{j}\left|\Psi_{n}\left(\vec{r}_{j}\right)\right|^{4}$ (where $\Psi_{n}$ is the n-th non-interacting eigenvector and $\Omega$ is the volume) by using the relation given in Ref. 18

$$
g=3(\pi(I-3))^{-1} \sum_{\mu}\left(\omega_{1} / \omega_{\mu}\right)
$$

where $\omega_{\mu}$ are the eigenvalues of the diffusion equation with the Neuman boundary condition for the stadium. It turns out that for a clean stadium or for small disorder $g$ acquires negative values which indicates that, although the energy spectrum follows RMT predictions, the eigenvectors are not yet fully random. Thus, in order to obtain a really random system we added two types of disorder: (i) on-site disorder, where the energy of each site is chosen randomly between $-W / 2$ and $W / 2$ and (ii) strong scatterers, where with a certain probability the site energy is set to zero or to a very large value (in our case $\epsilon_{j}=100 \mathrm{~V}$ ). 
In Fig. 2 we show the results for the spacing distribution for two different values of on-site disorder: $W=3 V$ corresponding to $g \sim 1$ and $W=V$ corresponding to $g \sim \infty$ (the conductance strongly increases when the value of $W$ decreases, but $W=V$ lies close to the region where Eq. (4) fails, thus we could not evaluate the exact value of $g$ but only asses, by extrapolation, that it is very large). We studied the case with (c,d) and without (a,b) a compensating background, and we built the distributions by averaging over the 20 realizations of disorder and over the number of electrons. From this figure we can clearly realize that by increasing the interaction strength $U$ we obtain a transition from a Wigner-Dyson surmise (solid thin line) to more symmetric and broad distributions which are well described by Gaussians (see for example the solid thick lines which fit the $U=1.5$ distributions). This behaviour is present for both strengths of disorder and for the different backgrounds. It is clearly seen that in the absence of a positive background the distributions for the same value of $g$ become broader, as predicted in Ref. [9]. This can be understood intuitively by noting that the electronic density in the presence of background is uniform, while in the absence of background there is a charge accumulation on the boundary (we have verified this directly from our calculation). Thus, without background the density is inhomogeneous and its effective value within the sample is lower, leading to a stronger influence of the interaction. The influence of $g$ is also clearly demonstrated in the figure. Smaller values of $g$ lead to a broader distribution for any kind of background.

A more quantitative representation can be obtained by calculating the distributions momenta. In Fig. 3 we show the dependence on the interaction strength of: (a) the average spacing $\left\langle\Delta_{2}\right\rangle$; (b) the standard deviation $\left\langle\delta \Delta_{2}\right\rangle \equiv \sqrt{\left\langle\left(\Delta_{2}-\left\langle\Delta_{2}\right\rangle\right)^{2}\right\rangle}$ and (c) the normalized deviation $\left\langle\delta \Delta_{2}\right\rangle /\left\langle\Delta_{2}\right\rangle$. In this figure we show the data corresponding to the values of parameter used in Fig. 2 and also the results obtained by using the strong scatterers disorder, where we distributed the scatterers randomly in the dot with a $5 \%$ probability. This allowed us to obtain a high value of the conductance in a case which is close to the clean case but where we can successfully use Eq. 4 .

Before discussing these results we need to asses their accuracy. The HF approximation 
is less and less accurate as the interaction strength increases. In fact we were not able to obtain reliable results, and not even convergence of the method, for $U / V$ greater or close to 2 , depending on the strength of the disorder. We realized that one of the first indicator of the lose of accuracy is the appearance of too large fluctuations in the addition spectrum. Thus to estimate the numerical errors affecting the results of Fig. [3 we fitted the distributions with a Gaussian function and we considered the long-tail deviations from the Gaussian as numerical errors. Naturally this procedure, which we used only for value of the $U / V$ larger than 0.5 , where the distributions have already lost the Wigner-like asymmetry of the noninteracting case, can hide some characteristics of the phenomenon. In fact we realized that the presence of the tails depends on the kind of disordered we used and that, for example, it is more pronounced in the clean dot case (not shown here) or in the strong scatterers disorder. We think that this effect could be related to the presence of scarred eigenstates of the system. These states in fact, for their strongly inhomogeneous charge distribution, can produce large charging fluctuations [19,20]. By considering the tails of the distribution as exclusively produced by the numerical approximation we obtained an overestimate of the error, but in all the cases shown in Fig. 3 we obtained error bars smaller or comparable with the size of the symbols we used, thus we decided not to show the error bars in the figures. Moreover this result confirms that at small values of the interaction the spacing distribution is already very close to a Gaussian.

As expected the average spacing (Fig. Ba) does not depend on the value of $g$. With the background $\left\langle\Delta_{2}\right\rangle$ is linear with $U$, as expected for a constant density system [10], while in the absence of the background the average spacing is somewhat suppressed because of the accumulation of charge on the boundary.

The standard deviation shows an enhancement as function of the interaction strength (Fig. 3b). The enhancement (compared to the theoretical RMT value of $\sqrt{4 / \pi-1} \sim 0.52 \Delta$, where $\Delta$ is the mean level spacing) strongly depends on the background and on $g$. The previously discussed role of the positive background in reducing the standard deviation is clearly seen. It is also clear that the standard deviation is smaller for larger values of $g$. 
In Fig. 3c we see that the normalized deviation for the cases in which a positive background exists saturates at values of $U \sim 1.5 \mathrm{~V}$. The normalized values saturate at values between $10 \%$ (for $g \rightarrow \infty$ ) and 20\% (for $g=1$ ) of the average spacing, in agreement with previous results [1]. Even for $g \rightarrow \infty$ the distribution seems to follow a Gaussian distribution, with an interaction independent width already at $U=1.5 \mathrm{~V}$ corresponding to an average $\bar{r}_{s}=0.7\left(\right.$ where $\bar{r}_{s}=\sqrt{\pi N}(U / 8 V) \int_{n_{1}}^{n_{2}} n^{-1 / 2} /\left(n_{2}-n_{1}\right)=\sqrt{\pi N}(U / 4 V)\left(\sqrt{n_{2}}-\sqrt{n_{1}}\right) /\left(n_{2}-n_{1}\right)$, with $n_{1}=20$ and $\left.n_{2}=120\right)$. In the absence of background the normalized deviation for the same value of $g$ is larger. Moreover, there is a minimum of the normalized deviation at $U \sim V$ after which this quantity starts growing. This is connected with the fact that at $U \sim V$ the average fluctuation $\left\langle\Delta_{2}\right\rangle$ starts to deviate from a linear dependence on $U$.

For the positive background case we are able to give an empirical scaling function for the standard deviation which describes rather well the influence of the dimensionless conductance $g$. From Fig. 3b it is clear that the standard deviation depends on $g$ and on the interaction strength $U$. In our model Hamiltonian we have five independent parameters: $W, U, V, n$ and $N$. Out of these parameters one may create a set of four independent dimensionless quantities $g, U / V, r_{s}$, and $\Delta / V$, and the standard deviation can be recast into the dimensionless form $\left\langle\delta \Delta_{2}\right\rangle^{*}=\left\langle\delta \Delta_{2}\right\rangle / \sqrt{4 / \pi-1} \Delta$. We observed that, by dividing the dimensionless deviation by $1+U / V \sqrt{g}$ all the curves of the same averaged electronic density seem to collapse on top of each other (see Fig. 4). This hints to a scaling of the dimensionless deviation $\left\langle\delta \Delta_{2}\right\rangle^{*}=(1+U / V \sqrt{g}) F\left(U / V, r_{s}\right)$, where $F\left(U / V, r_{s}\right)$ is some function of interaction strength and density. It is important to note that this scaling form does not agree with the expectations of perturbation theory. First, for $g \rightarrow \infty$ there should be no dependence on $U$ for weak interactions, which is clearly not the case. Also for a uniform distribution of charge one expects the corrections to the standard deviation to follow $1 / g$ [9] and not $1 / \sqrt{g}$. This $1 / \sqrt{g}$ dependence has been independently seen also in the work of Walker et. al. [12]. Of course one may blame these discrepancies on the HF approximation, but we note that for the relative weak interaction strength discussed here one expects HF to work rather well. One may also note that we are not able to recast the interplay between 
interaction strength and density as function of one combined parameter $r_{s}$. This may be the result of the high ratio of single electron spacing to the band width in our model.

In conclusions, the spacing distribution of spinless electrons in interacting quantum dots is Gaussian once $r_{s}$ is of order of one, independently of parameters such as dimensionless conductance and the positive background charge. Nevertheless, these parameters have a strong influence on the onset of the Gaussian distribution and its width. This might explain the considerable difference in the width of distribution seen between different experiments [1] [3], although it is not clear whether the physical parameters of the different dots are indeed different.

Useful discussions on the addition spectrum of quantum dots with O. Agam, B. L. Altshuler, A. Auerbach, Y. Gefen, C. M. Marcus, A. D. Mirlin, D. Orgad, O. Prus and U. Sivan are gratefully acknowledged. L.B. is grateful to G. Grosso and T. Wojta for useful suggestions about the Hartree-Fock method. We would like to thank the Israel Science Foundations Centers of Excellence Program for financial support. 


\section{FIGURES}

FIG. 1. The boundary condition used to obtain a chaotic dot without symmetries. In our calculations we used the following dimensions: $a=12, R=12, b=1$ which result in $N=265$.

FIG. 2. Distributions of the addition spectrum fluctuations. We show $P\left(\left(\Delta_{2}-e^{2} / C\right) /\left\langle\Delta_{2}-e^{2} / C\right\rangle\right)$ as function of the interaction strength $U$ using lines with different patterns. The solid thin line indicates the prediction of the RMT theory valid in the non-interacting case and the solid thick line a Gaussian fit of the $U=1.5 \mathrm{~V}$ case. The four graphs correspond to $W=1(\mathrm{a}, \mathrm{c})$ and $W=3(\mathrm{~b}, \mathrm{~d})$ cases without and with a positive background, respectively. The distributions are obtained averaging over 20 realizations of the on-site disorder and over the number of electrons ranging from $n=20$ to $n=120 . V=1$

FIG. 3. The average spacing $\left\langle\Delta_{2}\right\rangle$ (a), the standard deviation of the spacing $\left\langle\delta \Delta_{2}\right\rangle$ (b), and the normalized deviation $\left\langle\delta \Delta_{2}\right\rangle /\left\langle\Delta_{2}\right\rangle$ (c), as function of the interaction strength $U$ for different disordered stadia: $W=1$ (circles), $W=3$ (boxes), and $5 \%$ point scatterers (diamonds) with (dashed lines) and without background (solid lines). $V=1$

FIG. 4. The dimensionless deviation $\left\langle\delta \Delta_{2}\right\rangle^{*}$ (a) and $F\left(U / V, r_{s}\right)$ (b) as function of the interaction strength $U / V$ for different disordered stadia with background: $W=1$ (circles), $W=2$

(triangles), $W=3$ (boxes), and $5 \%$ point scatterers (diamonds). The empty symbols correspond to averaging over the interval $n=20 \div 120\left(\bar{r}_{s}=0.47 \mathrm{~V}\right)$ while the filled symbols to $n=20 \div 50\left(\bar{r}_{s}=0.6 \mathrm{~V}\right)$. The values of $g$ are shown in the labels. 


\section{REFERENCES}

[1] U. Sivan et. al., Phys. Rev. Lett. 77, 1123 (1996).

[2] F. Simmel, T. Heinzel and D. A. Wharam, Europhys. Lett. 38, 123 (1997).

[3] S. R. Patel et. al., Phys. Rev. Lett. 80, 4522 (1998).

[4] M. A. Kastner, Rev. Mod. Phys. 64, 849 (1992).

[5] U. Meirav and E. B. Foxman, Semicond. Sci. Technol. 10, 255 (1995).

[6] R. C. Ashoori, Nature 379, 413 (1996).

[7] P. L. McEuen, Science 278, 1729 (1997).

[8] R. Berkovits, Phys. Rev. Lett. 81,(1998).

[9] Ya. M. Blanter, A. D. Mirlin and B. A. Muzykantskii, Phys. Rev. Lett. 78, 2449 (1997).

[10] R. Berkovits and B. L. Altshuler, Phys. Rev. B 55, 5297 (1997).

[11] D. Organ and S. Levit (unpublished).

[12] P. N. Walker, Y. Gefen and G. Montambaux (unpublished).

[13] M. C. Gutzwiller, Chaos in classical and quantum mechanics, Springer-Verlag (1990).

[14] E.J. Heller, Phys. Rev. Lett. 53, 1515 (1984).

[15] R. Akis, J.P. Bird and D.K. Ferry, J. Phys. Condens. Matter 8, L667-L674 (1996).

[16] G. Bouzerar and D. Poilblanc, Phys. Rev. B52, 10772 (1995).

[17] F. Epperlein, M. Schreiber and T. Vojta, Phys. Rev. B56, 5890 (1997).

[18] V. N. Prigodin and B. L. Altshuler, Phys. Rev. Lett. 80, 1944 (1998).

[19] M. Stopa, Physica B 251228 (1998).

[20] L. Bonci and R. Berkovits, in preparation. 


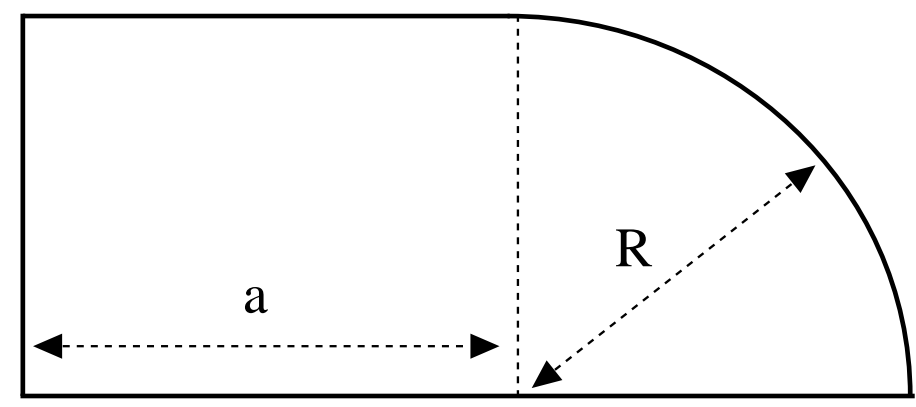

Fig.1 - Bonci et al. 


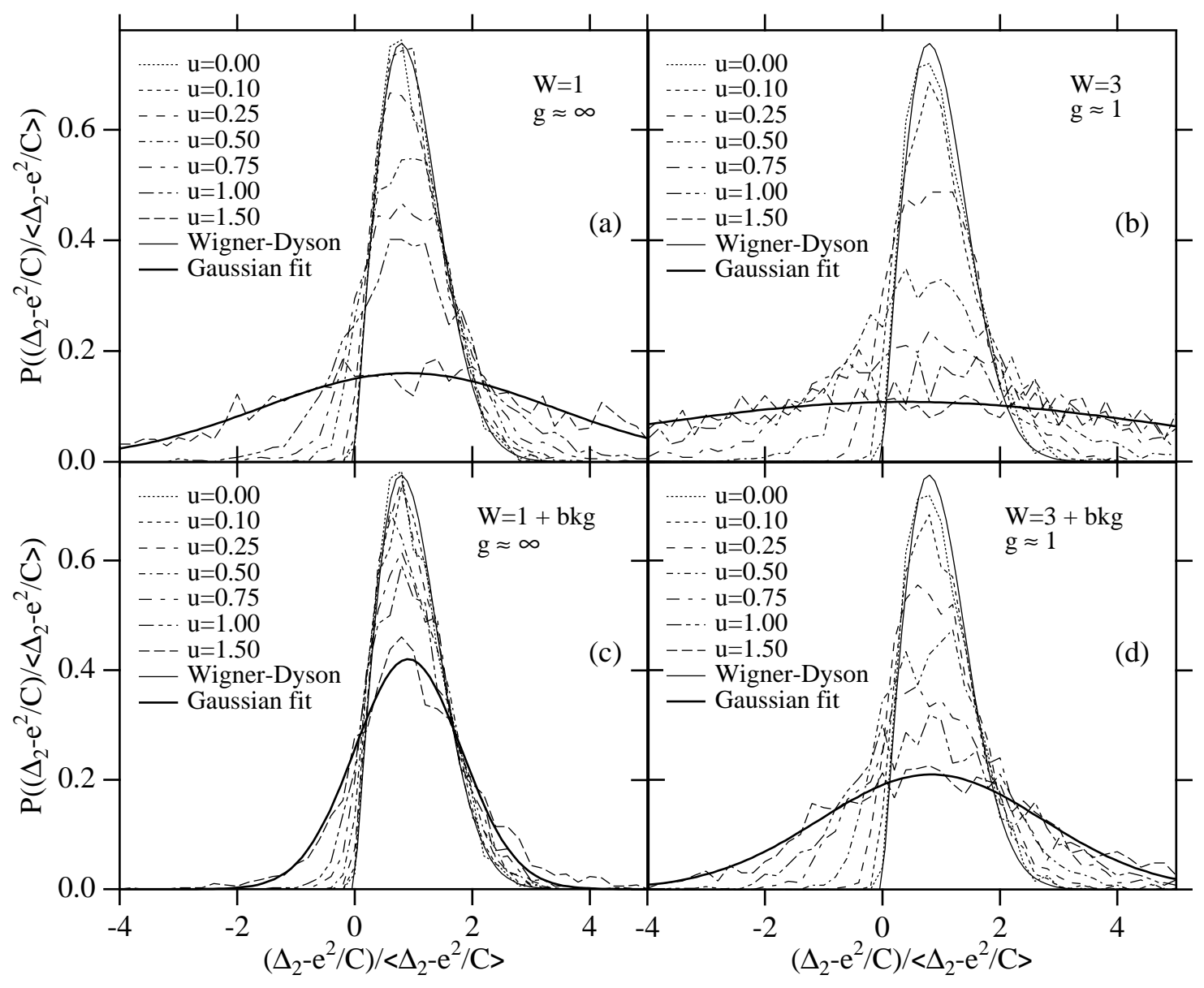

Fig. 2 - Bonci et al. 


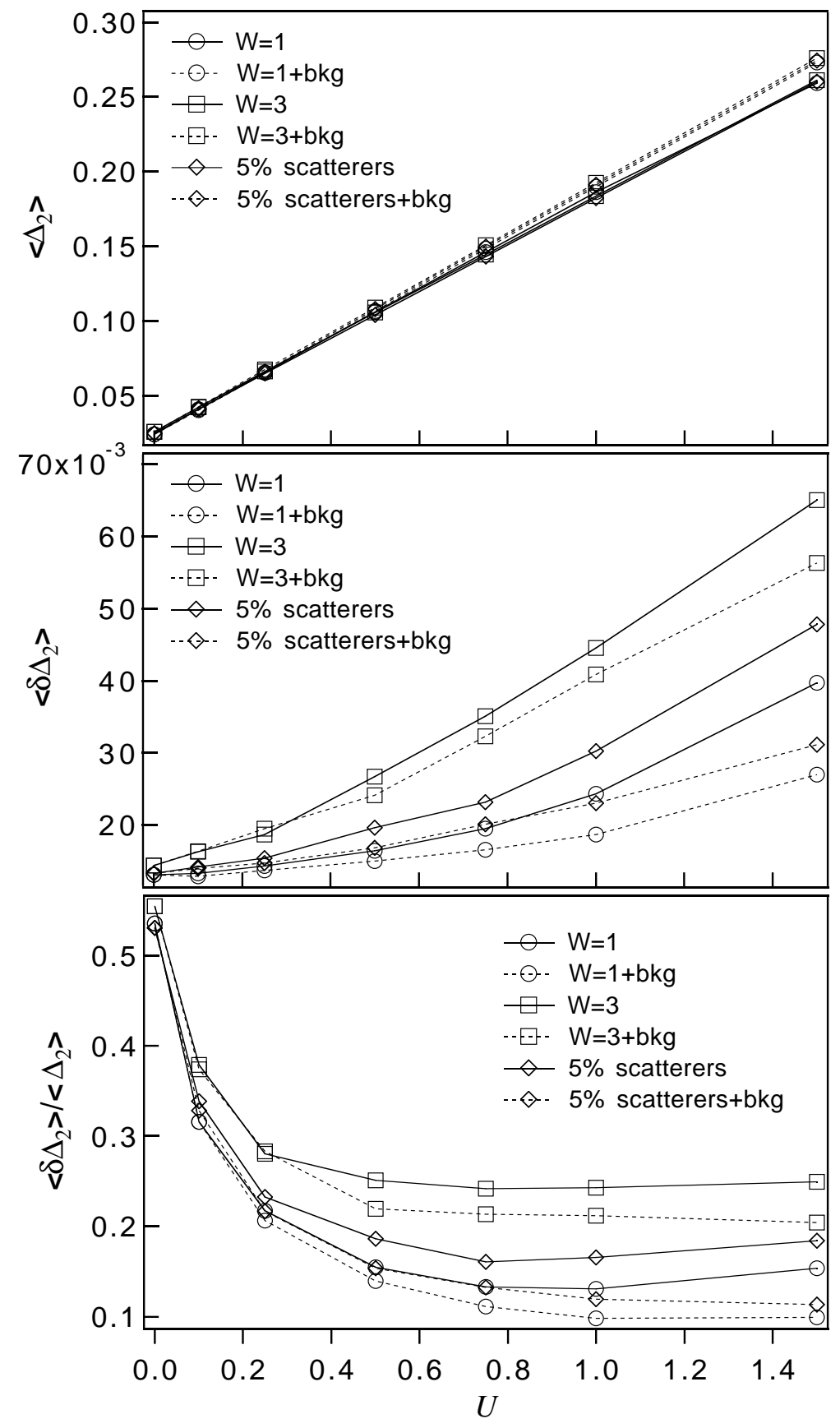

Fig. 3 - Bonci et al. 

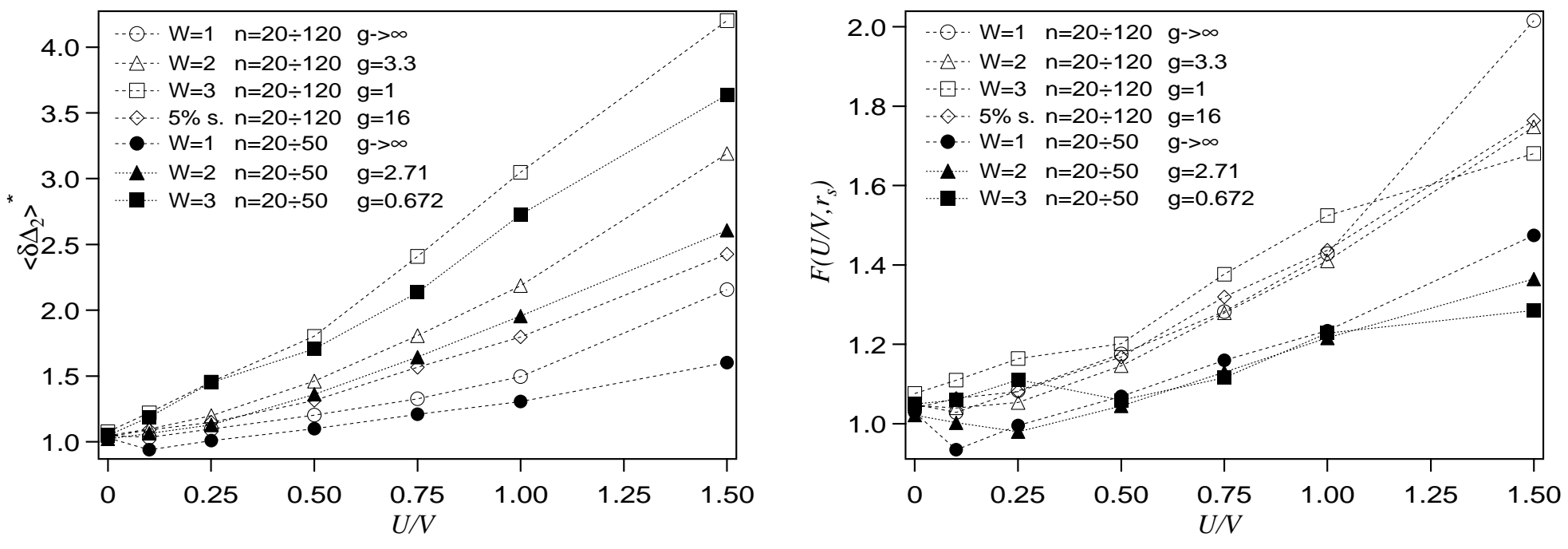

Fig. 4 - Bonci et al. 